\title{
QUASI-CONVERGENCE OF RICCI FLOW FOR A CLASS OF METRICS
}

\author{
RICHARD HAMILTON AND JAMES ISENBERG
}

\begin{abstract}
We show that for a certain family of Riemannian metrics on a twisted three-torus, the Ricci flow always asymptotically approaches that of a sub-family of locally homogeneous metrics.
\end{abstract}

\section{INTRODUCTION}

Converging Ricci flows have been found to be very useful for studying the relationship between manifold topologies and the metrics which they admit. For two-dimensional manifolds, one finds [1] [2] that all normalized Ricci flows converge; this provides a new (deformation-type) proof of the well-known two-dimensional uniformization theorem relating compact surfaces and constant curvature metrics. For three and four dimensional manifolds, normalized Ricci flows of positive curvature metrics converge to sphere metrics [3] [4]; one thereby determines that such metrics occur only on $\mathbb{S}^{3} / \Gamma$ and $\mathbb{S}^{4} / \Gamma$ (where $\Gamma$ denotes some finite group).

Ricci flows on manifolds of three or more dimensions do not generally converge, however, so to learn more about the relationship between topologies and metrics, it should be useful to study how non-converging Ricci flows behave. While the general non-converging Ricci flow is liable to involve various types of pinching singularities with unbounded curvature, that of many of the locally homogeneous metrics behave in a much quieter, though singular way [5]. The Ricci flow of a three-dimensional metric with the Heisenberg group for its isometry group, for example, slowly approaches the metric of a flat

This work has been partially supported by NSF grant DMS-90-0333 at the University of California, and NSF grant PHY-9012301 at the University of Oregon. 
two-dimensional torus, with its curvature approaching zero at the rate $1 / t$ as two of its directions expand while the other shrinks. The Ricci flows of many other locally homogeneous metrics behave similarly. (The $1 / t$ decay of the curvature is characteristic.)

While it may be that this quieter type of non-converging Ricci flow is peculiar to locally homogeneous geometries, it is also very possible that this sort of behavior occurs much more generally. In this paper, we provide some evidence for this. Specifically, we investigate the Ricci flow of a family of non-homogeneous metrics and show that for all of them, we have this sort of quiet, singular Ricci flow. In fact, we find that the Ricci flow for all metrics in this family asymptotically approaches the flow of a sub-family of locally homogeneous metrics which behaves much like the Heisenberg example noted above. We call this phenomenon "quasi-convergence" of the Ricci flow.

Most of this paper is devoted to proving this result. Before doing that, however, we define in $\S 1$ the family of metrics - the "solv-Gowdy metrics" which we wish to study, and we set up the Ricci flow equations for these metrics in $\S 2$. We solve these equations in $\S 3$ for those solv-Gowdy metrics which are locally homogeneous, thus obtaining in explicit form the Ricci flow for this sub-family, towards which the Ricci flow of all solv-Gowdy metrics will asymptotically approach. Finally in $\S 4$ we state the main theorem and carry out its proof. We make a few concluding remarks in $\S 5$.

\section{The Solv-Gowdy Metrics}

In previous work [6], one of us has studied the Ricci flow of a certain family of curvature-indefinite metrics on the three-torus. These metrics, which arise naturally in the study of cosmological solutions of Einstein's equations with $\mathrm{T}^{2}$ symmetry (the so-called "Gowdy spacetimes" [7]) take the form

$$
g=e^{A} d \theta^{2}+e^{f}\left[e^{W} d x^{2}+e^{-W} d y^{2}\right]
$$

where $A$ and $W$ are periodic functions of the coordinate $\theta$, and $f$ is a constant. One finds that this metric form is preserved under Ricci flow (with $A, W$ and $f$ as functions of $t$ ) and further one finds that for any initial choice of $A, W$ 
and $f$, the flow converges exponentially to some flat metric (i.e., one with $W$ independent of $\theta[6])$.

Here we wish to consider geometries whose metrics take essentially the same form (1.1), but live on a solv-twisted torus rather then on $\mathrm{T}^{3}$. A solv-twisted torus $\mathcal{T}_{\Lambda}^{3}$ is a $\mathrm{T}^{2}$ bundle over the circle, with the transition function taking values in $\mathrm{SL}(2, \mathbb{Z})$. A single $\mathrm{SL}(2, \mathbb{Z})$ matrix $\Lambda$ specifies a given solv-twisted torus, and if $\Lambda$ is chosen to be symmetric (and hence diagonalizable), then a diagonal metric of the form (1.1) - but with $A$ and $W$ non-periodic in $\theta$-is compatible with $\mathcal{T}_{\Lambda}^{3}$.

For our work here, it is useful to make a particular choice of the matrix $\Lambda$, and thereby fix the manifold $\mathcal{T}_{\Lambda}^{3}$. We shall choose $\Lambda=\left(\begin{array}{ll}2 & 1 \\ 1 & 2\end{array}\right)$. One readily verifies that a metric of the form (1.1) is compatible with $\mathcal{T}_{\left(\begin{array}{ll}2 & 1 \\ 1 & 2\end{array}\right)}^{3}$ as long as we require

$$
A(\theta+2 \pi)=A(\theta)
$$

and

$$
W(\theta+2 \pi)=W(\theta)+2 \ln \lambda
$$

where $\lambda=\frac{3+\sqrt{5}}{2}$ is the (larger) eigenvalue of $\Lambda$.

Note that these geometries $\left(\mathcal{T}_{\left(\begin{array}{ll}2 & 1 \\ 1 & 3\end{array}\right)}^{3}, g\right)$-which we shall call the "solv-Gowdy" family - do not have any isometries. However, they all possess a local $\mathrm{T}^{2}$ isometry; i.e. if $g$ is pulled back to the $\mathbb{R}^{3}$ cover of $\mathcal{T}_{\left(\begin{array}{l}2 \\ 1\end{array}\right)}^{3}$, the resulting geometry is $\mathrm{T}^{2}$ symmetric.

Note also that the solv-Gowdy geometries do not include any Einstein metrics ${ }^{1}$. Hence, Ricci flow confined to this family of geometries cannot converge.

\footnotetext{
${ }^{1}$ To see that there are no Einstein metrics among the solv-Gowdy geometries, we may write out the Einstein metric condition- $\operatorname{Ric}[g]=\mu g$-in terms of the functions $A$ and $W$ from eq. (1.1), and see that this condition holds iff $\mu=0$. But $\operatorname{Ric}[g]=0$ iff $W$ is constant, and this is incompatible with condition (1.2b).
} 


\section{Ricci Flow Equations}

Working with the arc-length coordinate

$$
s(\theta):=\int_{0}^{\theta} e^{A(u)} d u
$$

we calculate the non zero components for the metric (1.1) to be the following:

$$
\begin{aligned}
R_{\theta \theta} & =-\frac{1}{2} e^{A}\left(\frac{\partial}{\partial s} W\right)^{2} \\
R_{x x} & =-\frac{1}{2} e^{f+W} \frac{\partial^{2}}{\partial s^{2}} W \\
R_{y y} & =\frac{1}{2} e^{f-W} \frac{\partial^{2}}{\partial s^{2}} W
\end{aligned}
$$

Substituting these expressions into the (un-normalized) Ricci flow equation

$$
\frac{\partial}{\partial t} g_{i j}=-2 R_{i j}
$$

one readily verifies that the metric form (1.1) —and hence, the solv-Gowdy family - is preserved by the flow. We may now express the Ricci flow for this family of geometries in terms of evolution equations for the functions $W(\theta, t)$, $A(\theta, t)$, and $f(t)$ :

$$
\begin{aligned}
\frac{\partial}{\partial t} W & =\frac{\partial^{2}}{\partial s^{2}} W \\
\frac{\partial}{\partial t} A & =\frac{1}{2}\left(\frac{\partial}{\partial s} W\right)^{2} \\
\frac{\partial}{\partial t} f & =0
\end{aligned}
$$

The analysis of this system of partial differential equations is somewhat hampered by the fact that $W$ is not a continuous (periodic) function on the circle. Note, however, that $W$ itself does not appear on the right hand side of equations (2.4a)-(2.4c); we have only $\frac{\partial}{\partial s} W$ and $\frac{\partial^{2}}{\partial s^{2}} W$, so it seems reasonable to do our analytical work directly with $Z:=\frac{\partial}{\partial s} W$ instead of $W$, and obtain $W$ by integration at the end of the analysis. The variable $Z$ is continuous on $\mathbb{S}^{1}$; we may account for the condition $(1.2 \mathrm{~b})$ by requiring that

$$
\int_{\mathbb{S}^{1}} Z d s=2 \ln \left(\frac{3+\sqrt{5}}{2}\right) .
$$


To get the Ricci flow equation of evolution for $Z$, we need the commutator

$$
\left[\frac{\partial}{\partial t}, \frac{\partial}{\partial s}\right]=-\frac{1}{2} Z^{2} \frac{\partial}{\partial s}
$$

We then find

$$
\frac{\partial}{\partial t} Z=\frac{\partial^{2}}{\partial s^{2}} Z-\frac{1}{2} Z^{3}
$$

This equation, together with the commutator (2.6) and the integral condition (2.5) comprise a self-contained system, which is the focus of our analysis. Once the behavior of $Z$ has been determined, that of $W$ and $A$ may be obtained by integrating $\frac{\partial}{\partial s} W=Z$ and $\frac{\partial}{\partial t} A=\frac{1}{2} Z^{2}$. The variable $f$ does not change under Ricci flow.

It will be useful for later purposes to know the Ricci flow equation for a few quantities related to $Z$. These are easily calculated; we display them for convenience:

$$
\begin{aligned}
\frac{\partial}{\partial t}\left(Z^{2}\right) & =\frac{\partial^{2}}{\partial s^{2}}\left(Z^{2}\right)-2\left(\frac{\partial}{\partial s} Z\right)^{2}-Z^{4} \\
\frac{\partial}{\partial t}\left(\frac{\partial}{\partial s} Z\right) & =\frac{\partial^{2}}{\partial s^{2}}\left(\frac{\partial}{\partial s} Z\right)-2 Z^{2}\left(\frac{\partial}{\partial s} Z\right) \\
\frac{\partial}{\partial t}\left(\frac{\partial}{\partial s} Z\right)^{2} & =\frac{\partial^{2}}{\partial s^{2}}\left(\frac{\partial}{\partial s} Z\right)^{2}-2\left(\frac{\partial^{2} Z}{\partial s^{2}}\right)-4 Z^{2}\left(\frac{\partial Z}{\partial s}\right)^{2}
\end{aligned}
$$

We also find that if we define $L$ to be the length of the $x=0, y=0$ circle in a given solv-Gowdy metrix- $L:=\int_{\mathbb{S}^{1}} d s=\int_{0}^{2 \pi} e^{A(\theta)} d \theta$-then the evolution equation of $L$ under Ricci flow is given by

$$
\frac{d}{d t} L=\frac{1}{2} \int Z^{2} d s .
$$

The differential operator $\frac{\partial^{2}}{\partial s^{2}}=e^{-A} \frac{\partial}{\partial \theta} e^{-A} \frac{\partial}{\partial \theta}$ appears in many of these equations. Note that, so long as $A(\theta, t)$ is bounded for finite $t$, this operator serves as the Laplacian in our analysis.

\section{RicCi Flow of the}

\section{LoCAlly Homogeneous Solv-Gowdy Geometries}

A metric of the form (1.1) with constant $W$ is flat, and not compatible with the solv-twisted manifold $\mathcal{T}_{\left(\begin{array}{ll}2 & 1 \\ 1 & 2\end{array}\right)}^{3}$ we are considering here. However, we may 
choose $Z$ = constant; we obtain a sub-family of locally homogeneous solv-type geometries, with the group $E(1,1)$ acting transitively on the covering space geometries. We call this sub-family the "locally homogeneous solv-Gowdy" geometries.

Let $\mathcal{Z}(t):=Z(\theta, t)$ for $Z$ constant in $\theta$; the Ricci flow equation for these geometries in terms of $\mathcal{Z}(t)$ is

$$
\frac{d}{d t} \mathcal{Z}=-\frac{1}{2} \mathcal{Z}^{3}
$$

If we let $\zeta$ denote the value of $\mathcal{Z}$ at $t=0$, then we find that

$$
\mathcal{Z}(t)=\frac{1}{\sqrt{t+\frac{1}{\zeta^{2}}}}
$$

describes the evolution of $\mathcal{Z}$ for all $t>0$. Based on this result, along with the condition

$$
\mathcal{Z}(t) L(t)=2 \ln \left(\frac{3+\sqrt{5}}{2}\right)
$$

[which follows from condition (2.5)], we easily determine for these geometries

$$
\begin{aligned}
L(t) & =2 \ln \left(\frac{3+\sqrt{5}}{2}\right) \sqrt{t+\frac{1}{\zeta^{2}}} \\
e^{A(t)} & =\frac{1}{\pi} \ln \left(\frac{3+\sqrt{5}}{2}\right) \sqrt{t+\frac{1}{\zeta^{2}}}
\end{aligned}
$$

and

$$
W(\theta, t)=\frac{\theta}{\pi} \ln \left(\frac{3+\sqrt{5}}{2}\right)+\Omega
$$

where $\Omega$ is the value of $W$ at $\theta=0$ and $t=0$. The metric (1.1) now takes the form

$$
\begin{aligned}
g=\frac{\ln ^{2}\left(\frac{3+\sqrt{5}}{2}\right)}{\pi^{2}} & \left(t+\frac{1}{\zeta^{2}}\right) d \theta^{2} \\
+ & e^{F}\left[\left(\frac{3+\sqrt{5}}{2}\right)^{\theta / \pi} e^{\Omega} d x^{2}+\left(\frac{3+\sqrt{5}}{2}\right)^{-\theta / \pi} e^{-\Omega} d y^{2}\right]
\end{aligned}
$$

where $F$ is the (constant) value of $f$. 
Note, from (3.7), that the locally homogeneous solv-Gowdy geometries make up a three-parameter- $(S, F, \Omega)$-family. For any choice of these parameters, the Ricci flow of $g$ grows as $\sqrt{t}$ along the $\theta$-direction, and remains unchanged along the others ${ }^{2}$. If we calculate the curvature, we find that

$$
R_{\theta \theta}=-\frac{1}{2 \pi} \ln \left(\frac{3+\sqrt{5}}{2}\right) \frac{1}{\sqrt{1+\zeta^{2} t^{2}}}
$$

with all the other components vanishing. Hence, as the geometry expands, in the $\theta$ direction, the curvature gets flatter: We have

$$
R^{\alpha \beta} R_{\alpha \beta} \sim \frac{1}{t^{2}}
$$

\section{Main Theorem And Proof}

Our claim is that Ricci flow of any solv-Gowdy metric asymptotically approaches the Ricci flow of a locally homogeneous solv-Gowdy metric. Technically, we shall show this by proving that as the Ricci flow of any solv-Gowdy metric proceeds towards $t \rightarrow \infty$, the quantity $\frac{\partial}{\partial s} Z$ rapidly approaches zero, and the quantity $Z(\theta, t)$ behaves more and more like $\mathcal{Z}(t)$ for some locally homogeneous metric.

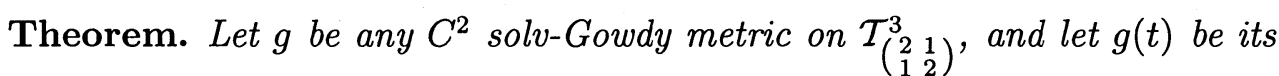
Ricci flow. There exists a positive constant $T$ such that, for $t \geq T$, the quantity $Z(\theta, t)$ corresponding to $g(t)$ satisfies the condition

$$
\left|\frac{\partial}{\partial s} Z(\theta, t)\right|<\frac{K}{(t-T)^{2}}
$$

where $K$ is a positive constant. As well, for $t>T, Z$ also satisfies the condition

$$
\frac{1}{\sqrt{(t-T)+\frac{1}{m^{2}}}} \leq Z(\theta, t) \leq \frac{1}{\sqrt{(t-T)+\frac{1}{M^{2}}}}
$$

where $m$ and $M$ are a pair of positive constants.

\footnotetext{
${ }^{2}$ In volume-normalized Ricci flow, these geometries would of course shrink along the $x$ and $y$ directions while it grows along the $\theta$-direction.
} 
Proof. Throughout this proof, we often wish to derive estimates for solutions of nonlinear parabolic equations-like (2.7) - based on the nonlinear terms in the equations. To do this, the following Lemma (which has been proved in [4]) can be very useful.

Lemma. Let $D$ be a compact set, and let the function $\psi: D \times \mathbb{R} \rightarrow \mathbb{R}$ be a smooth solution of a partial differential equation of the form

$$
\frac{\partial}{\partial t} \psi=F[\psi, x, t]
$$

where $F$ is a functional of $\psi$, of $x \in D$ and of $t \in \mathbb{R}$. Let $M(t)$ be a solution of the ordinary differential equation

$$
\frac{d}{d t} M=E[M, t]
$$

where $E$ is a function of $M$ and of $t \in \mathbb{R}$ which satisfies the condition

$$
E[M, t] \geq \operatorname{Sup}_{\operatorname{Max}_{D}[\psi]}(F[\psi, x, t]) \text { for } M \geq \operatorname{Max}_{D}[\psi] ;
$$

and let $m(t)$ be a solution of the ordinary differential equation

$$
\frac{d}{d t} m=G[m, t]
$$

where $G$ is a function of $m$ and $t \in \mathbb{R}$ which satisfies the condition

$$
G[m, t] \geq \operatorname{Inf}_{\operatorname{Min}_{D}[\psi]}(F[\psi, x, t]) \text { for } m \leq \operatorname{Min}_{D}[\psi]
$$

(Here, “ $\operatorname{Sup}_{\operatorname{Max}_{D}[\psi]}(F[\psi, x, t]) "$ means that we are to find the least upper bound of $F$ evaluated at all $x$ and $\psi(x)$ for which $\psi(x)=\operatorname{Max}_{D}[\psi]$; similarly for “ $\operatorname{Inf}_{\operatorname{Min}_{D}[\psi]}(F[\psi, x, t]) "$.)

Then if $M(0) \geq \operatorname{Max}_{D}[\psi(x, 0)]$ and if $m(0) \leq \operatorname{Min}_{D}[\psi(x, 0)]$, we have

$$
M \geq \operatorname{Max}_{D}[\psi(x, t)]
$$

and

$$
m \leq \operatorname{Min}_{D}[\psi(x, t)]
$$

for all $t$. 
The proof of the theorem proceeds via four main steps. In Step 1, we establish certain preliminary time-dependent bounds for the quantities $|Z|$, $\left|\frac{\partial}{\partial s} Z\right|$, and $L$. In Step 2, we use the bounds to show that there exists a $T>0$ such that for $t \geq T, Z(\theta, t)$ is everywhere positive. In Step 3, we use the Lemma and the positivity of $Z(\theta, t)$ to establish the inequality (4.2), and finally in Step 4, we use inequality (4.2) and the Lemma to show that (4.1) holds true.

Step 1. (Estimates for $|Z|, L$, and $\left|\frac{\partial}{\partial s} Z\right|$ ) $|Z|$.

Letting $F[Z, x, t]=-\frac{1}{2} Z^{3}$ we may apply the Lemma to eq. (2.7); we consequently find that for $t \geq 0$,

$$
\begin{aligned}
|Z(\theta, t)| & \leq \frac{1}{\sqrt{t+\frac{1}{\nu^{2}}}} \\
& <\frac{1}{\sqrt{t}}
\end{aligned}
$$

where $\nu:=\operatorname{Max}_{\mathbb{S}^{1}}|Z(\theta, 0)|$. This tells us that whatever $Z(\theta, 0)$ is-and it can be any $C^{1}$ function for which $\int Z d s=2 \ln \left(\frac{3+\sqrt{5}}{2}\right)$-by $t=1$ we must have $|Z(\theta, 1)| \leq 1$.

$L$.

Based on eqs. (2.9) and (4.3), we find that

$$
\begin{aligned}
\frac{d}{d t} L & =\int \frac{1}{2} \operatorname{Max}_{\mathbb{S}^{1}} Z^{2} d s \\
& \leq \frac{1}{2} Z^{2} L \\
& \leq \frac{1}{2\left(t+\frac{1}{\nu^{2}}\right)} L .
\end{aligned}
$$

This inequality implies (recall that $t \geq 0$ and $L \geq 0$ ) that

$$
\frac{d}{d t}\left(\frac{L}{\sqrt{t+\frac{1}{\nu^{2}}}}\right) \leq 0
$$


from which we obtain an upper ( $t$-dependent) bound on $L$ :

$$
L(t) \leq \sqrt{t+\frac{1}{\nu^{2}}} \lambda \nu
$$

where $\lambda:=L(0)$.

To get a lower ( $t$-dependent) bound on $L$, recall from eq. (2.5) that $\int_{\mathbb{S}^{1}} Z d s=$ $2 \ln \left(\frac{3+\sqrt{5}}{2}\right)$. Using eq. (4.3), we find that

$$
\begin{aligned}
\left|\int_{\mathbb{S}^{1}} Z d s\right| & \leq \int_{\mathbb{S}^{1}}|Z| d s \\
& \leq \frac{1}{\sqrt{t+\frac{1}{\nu^{2}}}} L .
\end{aligned}
$$

Combining (2.5) and (4.7), we find

$$
L(t) \geq \sqrt{t+\frac{1}{\nu^{2}}} 2 \ln \left(\frac{3+\sqrt{5}}{2}\right)
$$

which is the desired lower bound.

Since we are mainly interested in large $t$ behavior, we may rewrite these upper and lower bounds for $t \geq 1$ in the form

$$
2 \ln \left(\frac{3+\sqrt{5}}{2}\right) \leq \frac{L(t)}{\sqrt{t}} \leq \bar{\lambda}
$$

where $\bar{\lambda}:=L(1)$.

$\left|\frac{\partial}{\partial s} Z\right|$.

Our first estimate for $\left|\frac{\partial}{\partial s} Z\right|$ can be obtained by using an argument of the type developed by Shi [8]; it goes as follows: Combining eqs. (2.8a) and (2.8c), we obtain the following evolution equation

$$
\begin{aligned}
& \frac{\partial}{\partial t}\left[2 t\left(\frac{\partial}{\partial s} Z\right)^{2}+Z^{2}\right] \\
& \quad=\frac{\partial^{2}}{\partial s^{2}}\left[2 t\left(\frac{\partial}{\partial s} Z\right)^{2}+Z^{2}\right]-Z^{2}-4 t\left\{\left(\frac{\partial^{2}}{\partial s^{2}} Z\right)^{2}+2 Z^{2}\left(\frac{\partial}{\partial s} Z\right)^{2}\right\}
\end{aligned}
$$


which implies the inequality

$$
\frac{\partial}{\partial t}\left[2 t\left(\frac{\partial}{\partial s} Z\right)^{2}+Z^{2}\right] \leq \frac{\partial^{2}}{\partial s^{2}}\left[2 t\left(\frac{\partial}{\partial s} Z\right)^{2}+Z^{2}\right]
$$

As noted earlier, $\frac{\partial^{2}}{\partial s^{2}}=e^{-A} \frac{\partial}{\partial \theta} e^{-A} \frac{\partial}{\partial \theta}$. So, recalling the Ricci flow equation (2.4b) for $A-\frac{\partial}{\partial t} A=\frac{1}{2} Z^{2}$-we see from the estimate (4.3) on $|Z|$ that $A$ is finite for finite $t$, and hence $\frac{\partial^{2}}{\partial s^{2}}$ is a non degenerate elliptic operator for finite $t$. Thus we may apply the Maximum Principle to eq. (4.11) and infer from it that

$$
\begin{aligned}
\left.\operatorname{Max}_{\mathbb{S}^{1}}\left[2 t\left(\frac{\partial}{\partial s} Z\right)^{2}+Z^{2}\right]\right|_{t \geq 0} & \leq\left.\operatorname{Max}_{\mathbb{S}^{1}}\left[2 t\left(\frac{\partial}{\partial s} Z\right)^{2}+Z^{2}\right]\right|_{t=0} \\
& =\nu^{2}
\end{aligned}
$$

A bit of algebra then produces the estimate

$$
\left|\frac{\partial}{\partial s} Z\right| \leq \frac{\nu}{\sqrt{2 t}}
$$

While inequality (4.13) is encouraging, it only guarantees that $\left|\frac{\partial}{\partial s} Z\right|$ decays at the same rate as $|Z|$. We need to show that the decay of $\left|\frac{\partial}{\partial s} Z\right|$ is much faster. We get a somewhat faster decaying using the following argument:

We wish to estimate $\left|\frac{\partial}{\partial s} Z\right|$ at some fixed positive value of $t$ which we shall call $\tau$. So, let us consider the quantity $\left[2\left(t-\frac{\tau}{2}\right)\left(\frac{\partial}{\partial s} Z\right)^{2}+Z^{2}\right]$ and calculate its $t$-derivative; we get

$$
\begin{aligned}
& \frac{\partial}{\partial t}\left[2\left(t-\frac{\tau}{2}\right)\left(\frac{\partial}{\partial s} Z\right)^{2}+Z^{2}\right]=\frac{\partial^{2}}{\partial s^{2}}\left[2\left(t-\frac{\tau}{2}\right)\left(\frac{\partial}{\partial s} Z\right)^{2}+Z^{2}\right] \\
&-4\left(t-\frac{\tau}{2}\right)\left\{\left(\frac{\partial^{2}}{\partial s^{2}} Z\right)^{2}+2 Z^{2}\left(\frac{\partial}{\partial s} Z\right)^{2}\right\}-Z^{4}
\end{aligned}
$$

Now for $t>\frac{\tau}{2}$, eq. (4.14) implies the inequality

$$
\frac{\partial}{\partial t}\left[2\left(t-\frac{\tau}{2}\right)\left(\frac{\partial}{\partial s} Z\right)^{2}+Z^{2}\right] \leq \frac{\partial^{2}}{\partial s^{2}}\left[2\left(t-\frac{\tau}{2}\right)\left(\frac{\partial}{\partial s} Z\right)^{2}+Z^{2}\right]
$$


It then follows from the Maximum Principle that

$$
\begin{aligned}
\left.\operatorname{Max}_{\mathbb{S}}\left[2\left(t-\frac{\tau}{2}\right)\left(\frac{\partial}{\partial s} Z\right)^{2}+Z^{2}\right]\right|_{t \geq \frac{\tau}{2}} & \leq\left.\operatorname{Max}_{\mathbb{1}^{1}}\left[2\left(t-\frac{\tau}{2}\right)\left(\frac{\partial}{\partial s} Z\right)^{2}+Z^{2}\right]\right|_{t=\frac{\tau}{2}} \\
& =\operatorname{Max}_{\mathbb{S}^{1}} Z^{2}\left(\theta, \frac{\tau}{2}\right) .
\end{aligned}
$$

From (4.3), we know that

$$
Z^{2}\left(\theta, \frac{\tau}{2}\right) \leq \frac{2}{\tau}
$$

so if we combine inequalities (4.16) and (4.17), we have

$$
\left|\frac{\partial}{\partial s} Z(\theta, \tau)\right| \leq \frac{\sqrt{2}}{\tau}
$$

This argument works for any choice of $t=\tau>0$, so we have

$$
\left|\frac{\partial}{\partial s} Z(\theta, t)\right| \leq \frac{\sqrt{2}}{t} \text {. }
$$

While this $\frac{1}{t}$ decay rate for $\left|\frac{\partial}{\partial s} Z(\theta, t)\right|$ is faster than that which we have obtained for $|Z(\theta, t)|$, it could be argued, using scaling arguments, that is does not necessarily indicate asymptotic approach to a locally homogeneous Ricci flow. So we will prove in Step 4 that $\left|\frac{\partial}{\partial s} Z(\theta, t)\right|$ has a faster rate of decay.

Step 2. (Positive Definiteness of $Z$ )

One may choose $g$ at $t=0$ so that $Z(\theta, 0)$ is any $C^{1}$ function from the circle to the reals, as long as the condition $\int_{\mathbb{S}^{1}} Z d s=2 \ln \left(\frac{3+\sqrt{5}}{2}\right)$ is satisfied. In particular, $Z(\theta, 0)$ may have zeroes, and it may be negative for some $\theta$.

If, however, as the Ricci flow progresses, $g(t)$ is supposed to asymptotically approach a locally homogeneous Ricci flow with $Z(\theta, t)$ constant in $\theta$, then one expects that there exists some $T>0$ such that for $t>T, Z(\theta, t)$ is positive definite. We show this here, via a pair of claims.

Claim A. If $Z(\theta, T)>0$, then $Z(\theta, t)>0$ for all $t>T$. 
Proof of Claim A. Since the domain of $Z(\theta, T)$ is compact, and since $Z(\theta, T)$ is $C^{1}$, there exists a positive number $\mu$ such that $Z(\theta, T)>\mu$. It now follows from the Lemma that

$$
Z(\theta, t) \geq \frac{1}{\sqrt{(t-T)+\frac{1}{\mu^{1}}}}
$$

for $t \geq T$. Thus, for $t>T, Z(\theta, t)$ is positive.

Claim B. There exists $T>0$ such that $Z(\theta, T)>0$.

Proof of Claim B. Say $Z(\theta, 1)$ is zero for one or more values of $\theta$. [ If $Z(\theta, 1)$ has no zeroes, then it follows from the continuity of $Z(\theta, 1)$ and from the condition $\int_{\mathbb{S}^{1}} Z d s=2 \ln \left(\frac{3+\sqrt{5}}{2}\right)$ that $Z(\theta, 1)>0$.] We shall show that if $Z(\theta, t)$ continues to have zeroes for all $t \in[1, \tau]$, then $\tau$ must be finite.

Let $t \in[1, \tau]$ and consider the quantity

$$
\Phi(t):=\int_{\mathbb{S}^{1}}\left(1-t Z^{2}(\theta, t)\right) d s
$$

Since we know from Step 1 that $|Z(\theta, t)|<\frac{1}{\sqrt{t}}$, we see that the integrand $1-t Z^{2}$ of $\Phi(t)$ is everywhere positive or zero. Further, since we also know from Step 1 that $\left|\frac{\partial}{\partial s} Z(\theta, t)\right|<\frac{\sqrt{2}}{t}$, it follows from the Mean Value Theorem that for all $\theta$ within arc length $\sigma$ of some zero of $Z(\theta, t)$, we have

$$
|Z(\theta, t)|<\frac{\sqrt{2}}{t} \sigma
$$

Now let $\mathcal{S}$ be an interval subset of $\mathbb{S}^{1}$ on which $t Z^{2} \leq \frac{1}{2}$. From (4.22), we see that as long as $L(t)>\frac{\sqrt{t}}{2}$, we may always choose $\mathcal{S}$ so that its length is at least $\frac{\sqrt{t}}{2}$. Since we have already determined in Step 1 that $L(t) \geq 2 \ln \left(\frac{3+\sqrt{5}}{2}\right) \sqrt{t}>$ 
$\frac{\sqrt{t}}{2}$, such a choice of $\mathcal{S}$ may always be made. We may thus calculate

$$
\begin{array}{rlrl}
\Phi(t) & =\int_{\mathbb{S}^{1}}\left(1-t Z^{2}\right) d s & \\
& \geq \int_{\mathcal{S}}\left(1-t Z^{2}\right) d s & & \text { since } 1-t Z^{2} \geq 0 \text { on } \mathbb{S}^{1} \\
& \geq \frac{1}{2} \int_{\mathcal{S}} d s & & \text { since } t Z^{2} \leq \frac{1}{2} \text { on } \mathcal{S} \\
& =\frac{1}{2} \text { length of } \mathcal{S} & \\
& \geq \frac{1}{4} \sqrt{t} & & \text { from above argument }
\end{array}
$$

By assumption, this inequality (4.23) holds for all $t \in[1, \tau]$.

If we divide inequality (4.23) by $t$ and rearrange its terms, we find that

$$
\int_{\mathbb{S}^{1}} Z^{2} d s \leq \frac{1}{t} L(t)-\frac{1}{4} \frac{1}{\sqrt{t}}
$$

Combining this result with the Ricci flow eq. (2.9) for $L(t)$, we have

$$
\frac{d}{d t} L \leq \frac{1}{2 t} L-\frac{1}{8} \frac{1}{\sqrt{t}}
$$

which implies (after multiplying through by $\frac{1}{\sqrt{t}}$ )

$$
\frac{d}{d t}\left(\frac{L}{\sqrt{t}}\right) \leq-\frac{1}{8 t} \text {. }
$$

We now integrate both sides of inequality (4.26) over $t$, from $t=1$ to $t=\tau$; we obtain

$$
\frac{L(\tau)}{\sqrt{\tau}}-L(1) \leq-\frac{1}{8} \ln (\tau)
$$

or

$$
\ln (\tau) \leq 8\left[L(1)-\frac{L(\tau)}{\sqrt{\tau}}\right] .
$$

Since $L(\tau) \geq 2 \ln \left(\frac{3+\sqrt{5}}{2}\right) \sqrt{\tau}$, inequality (4.28) tells us that

$$
\ln (\tau) \leq 8\left[L(1)-2 \ln \left(\frac{3+\sqrt{5}}{2}\right)\right] .
$$

The right hand side of inequality (4.29) is constant. Hence, it follows that $\ln (\tau)$ - and therefore $\tau$ itself-is bounded from above. We thus see that for 
some value of $T$ (no bigger than $\left.\frac{2^{16}}{(3+\sqrt{5})^{16}} e^{8 L(1)}\right), Z(\theta, t)$ must be nonzero-and thus positive-for all $t>T$.

Step 3. (Verification of Inequality (4.2))

Once we know that for some $T>0$ we have $Z(\theta, T)$ positive definite and hence bounded between a minimum $m$ and a maximum $M$, inequality (4.2) is a direct corollary of the Lemma.

Step 4. (Verification of Inequality (4.1))

In $\S 2$, we noted that the Ricci flow equation for $\Lambda:=\left(\frac{\partial}{\partial s} Z\right)^{2}$ is

$$
\frac{\partial}{\partial t} \Lambda=\frac{\partial^{2}}{\partial s^{2}} \Lambda-2\left(\frac{\partial^{2}}{\partial s^{2}} Z\right)^{2}-4 Z^{2} \Lambda
$$

We wish to obtain a $t$-dependent upper bound on $\Lambda$ by applying the Lemma to eq. $\left(2.8 c^{\prime}\right)$. So we need to examine

$$
\operatorname{Sup}_{\left(\operatorname{Max}_{\mathrm{S} 1}[\Lambda]\right)}\left[\frac{\partial^{2}}{\partial s^{2}} \Lambda-2\left(\frac{\partial^{2}}{\partial s^{2}} Z\right)^{2}-4 Z^{2} \Lambda\right]
$$

Since $-2\left(\frac{\partial^{2}}{\partial s^{2}} Z\right)^{2} \leq 0$ and since $\frac{\partial^{2}}{\partial s^{2}} \Lambda \leq 0$ for maximum values of $\Lambda$, we have

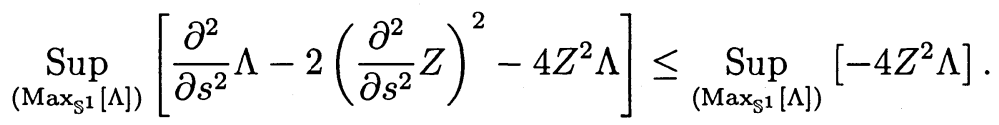

Using the lower bound for $Z(\theta, t)$ which we derived in Step 3, we have

$$
\operatorname{Sup}_{\left.\operatorname{Max}_{\mathbb{S}^{1}}[\Lambda]\right)}\left[-4 Z^{2} \Lambda\right] \leq\left(\frac{-4}{(t-T)+\frac{1}{m^{2}}}\right) \operatorname{Max}_{\mathbb{S}^{1}}[\Lambda] .
$$

Hence we are led to study the ordinary differential equation

$$
\frac{d}{d t} \xi=\left(\frac{-4}{(t-T)+\frac{1}{m^{2}}}\right) \xi
$$

This equation has the unique solution

$$
\xi(t)=\frac{\xi(T)}{\left[1+m^{2}(t-T)\right]^{4}}
$$


It thus follows from the Lemma that if $\eta$ denotes the value of $\operatorname{Max}_{\mathbb{S}^{1}}\left|\frac{\partial}{\partial s} Z\right|$ at $t=T$, then for $t>T$,

$$
\begin{aligned}
\left|\frac{\partial}{\partial s} Z(\theta, t)\right| & \leq \frac{\eta}{\left[1+m^{2}(t-T)\right]^{2}} \\
& \leq \frac{C}{(t-T)^{2}}
\end{aligned}
$$

where $C$ is the constant $\frac{\eta}{m^{2}}$. We thus verify inequality (4.1), and complete the proof of the theorem.

Why do we believe that the decay estimate (4.35) indicates asymptotic approach of the Ricci flow of the solv-Gowdy metrics to that of the locally homogeneous solv-Gowdy metrics, while the $\frac{1}{\sqrt{t}}$ and $\frac{1}{t}$ decay estimate obtained earlier are insufficient? We have seen (Step 3 of the proof) that $Z(\theta, t)$ decays towards zero at the rate $\frac{1}{\sqrt{t}}$ and no faster. Standard scaling type arguments can then be used to argue that $\left|\frac{\partial}{\partial s} Z(\theta, t)\right|$ should decay as $\frac{1}{t}$, whether there is asymptotic approach or not. The rate of decay contained in $(4.35)-\frac{C}{(t-T)^{2}}$-is faster than $\frac{1}{t}$ for large $t$, so we claim it verifies asymptotic approach.

\section{Conclusion}

Our result is, we believe, the first demonstration that the Ricci flow of a nontrivial family of metrics which cannot converge, nevertheless asymptotically approaches the Ricci flow of a family of locally homogeneous metrics. Clearly it would be useful to study whether this behavior occurs in more general families of metrics. Some studies of this nature are underway (e.g. with families of metrics constructed as warped products of a circle over general compact surfaces), and the indications are that this quasi-convergent-type behavior is present.

These studies are, however, very far from comprehensive. In all cases thus far examined, topological and symmetry restrictions have been built in to preclude (or at least discourage) the development of curvature singularities. Eventually, one must deal with the occurrence of such singularities. For now, 
we are making slow but finite progress toward understanding the behavior of nonsingular Ricci flow of three dimensional metrics.

\section{REFERENCES}

1. Hamilton, R., The Ricci flow on surfaces, in Mathematics and General Relativity, J. Isenberg ed., Contemp. Math. 71, Amer. Math. Soc. 1988.

2. Chow, B., The Ricci-Hamilton flow on the 2-sphere, J. Diff. Geom. (1990).

3. Hamilton, R., Three-manifolds with positive Ricci curvatures, J. Diff. Geom. 17 (1982), 255-306.

4. Hamilton, R., Four-manifolds with positive curvature operator, J. Diff. Geom. 27 (1986), 153-179.

5. Isenberg, J. and Jackson, M., Ricci flow of locally homogeneous geometries on closed manifolds, J. Diff. Geom. 35 (1992), 723-741.

6. Carfora, M., Isenberg, J. and Jackson, M., Convergence of the Ricci flow for metrics with infinite Ricci curvature, J. Diff. Geom. 31 (1990), 249-263.

7. Gowdy, R., Vacuum spacetimes with two-parameter spacelike isometry groups and compact invariant hypersurfaces: topology and boundary conditions, Ann. Phys. (USA) 83 (1974), 203-241.

8. Shi, W.-X., Deforming the metric on complete Riemannian manifolds, J. Diff. Geom. 30 (1989), 223-301.

University of California, San Diego, U. S. A.

UNIVERSITY OF OREgon, U. S. A.

ReCeived May 13, 1993. 